\title{
VARIABLE, OPTICALLY THICK, HOT PLASMA OBSERVED IN INTERACTING BINARIES*
}

\author{
Y. KONDO \\ Laboratory for Astronomy and Solar Physics, NASA-Goddard Space Flight Center, Greenbelt, Md., U.S.A. \\ G. E. McCLUSKEY \\ Division of Astronomy, Department of Mathematics, Lehigh University, Bethlehem, Pa., U.S.A. \\ and \\ S. B. PARSONS \\ Space Telescope Science Institute, Johns Hopkins University, Baltimore, Md., U.S.A.
}

(Received 5 August, 1983)

\begin{abstract}
We report recent International Ultraviolet Explorer (IUE) observations of two interacting binaries, R Arae and HD 207739. The ultraviolet spectra indicate the presence of optically-thick, variable hot plasma in those binary systems. These two binaries may belong to a class of binaries that are currently undergoing a rarely observed and probably short-lived phase in their evolution. Their properties are compared with those of two other interacting binaries, $\mathrm{U}$ Cephei and $\beta$ Lyrae.
\end{abstract}

\section{Introduction}

One of the important characteristics of interacting binary systems is the presence of gas, often a high temperature plasma within and/or surrounding the entire system and in the form of streams, disks, circumstellar rings, circumbinary shells, winds, hot spots, etc. The physical properties of this gas are affected by the binary nature of the system in which it originates. The signature of this gas may be observed photometrically or spectroscopically.

Here we report on two binaries which were recently observed with the International Ultraviolet Explorer (IUE) satellite observatory. The data indicate that these systems, R Arae (McClusky and Kondo, 1983) and HD 207739 (Parsons et al., 1983), may belong to a class of binaries that are currently undergoing a rarely observed and probably short-lived phase in their evolution. Their properties will be compared with two other binaries, which have also been studied in the ultraviolet, $\mathrm{U}$ Cephei and $\beta$ Lyrae.

\section{Discussion}

The orbital period of $R$ Arae (B9Vp + ?) is about 4.4 days (Sahade, 1952) and that of HD 207739 (F8II + ?) is about 18.6 days. Additional IUE high-resolution spectra

\footnotetext{
* Paper presented at the Lembang-Bamberg IAU Colloquium No. 80 on 'Double Stars: Physical Properties and Generic Relations', held at Bandung, Indonesia, 3-7 June, 1983.
} 
obtained since the completion of the two papers referred to above show strong evidence of the presence of a variable, optically thick, hot plasma which gives rise to nonmonotonic changes in the spectral energy distribution.

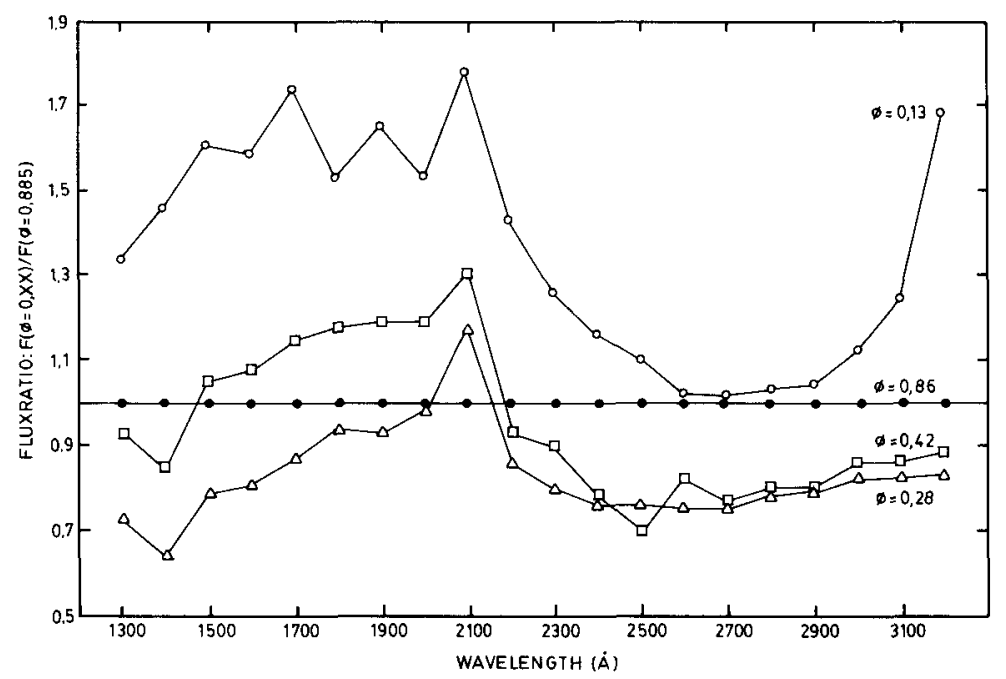

Fig. 1. IUE continuum variations of $\mathrm{R}$ Arae.

Figure 1 shows the spectral energy distribution of $R$ Arae at several phases. These curves are normalized to the distribution at phase 0.86 since this particular normalization yields curves of roughly the same shape. Figure 2 shows the spectral energy curves of HD 207739 at two phases, one phase arbitrarily normalized to the other. A similar spectral energy variation of $\mathbf{R}$ Arae was first observed with the Astronomical Netherlands Satellite (ANS) (Kondo et al., 1981).

If these spectral energy variations were simply the result of a temperature change of the plasma involved, the result would be a monotonic increase or decrease of the spectral gradient toward short wavelengths. Instead we observe an irregular increase in the ultraviolet flux at some wavelengths and a decrease at other wavelengths. These nonmonotonic variations in the spectral energy curve may occur if two or more regions of the plasma at different temperatures are of variable volume and/or temperature. The most striking variations occur in the continuum levels but the equivalent widths and profiles of a number of spectral lines also vary; in a few cases lines are not detectable at some phases and quite prominent at others. In order for the continuum to change so dramatically, the optical thickness of the plasma clouds involved must be substantially greater than unity. If the mass flow rate is near the conventionally quoted supercritical 


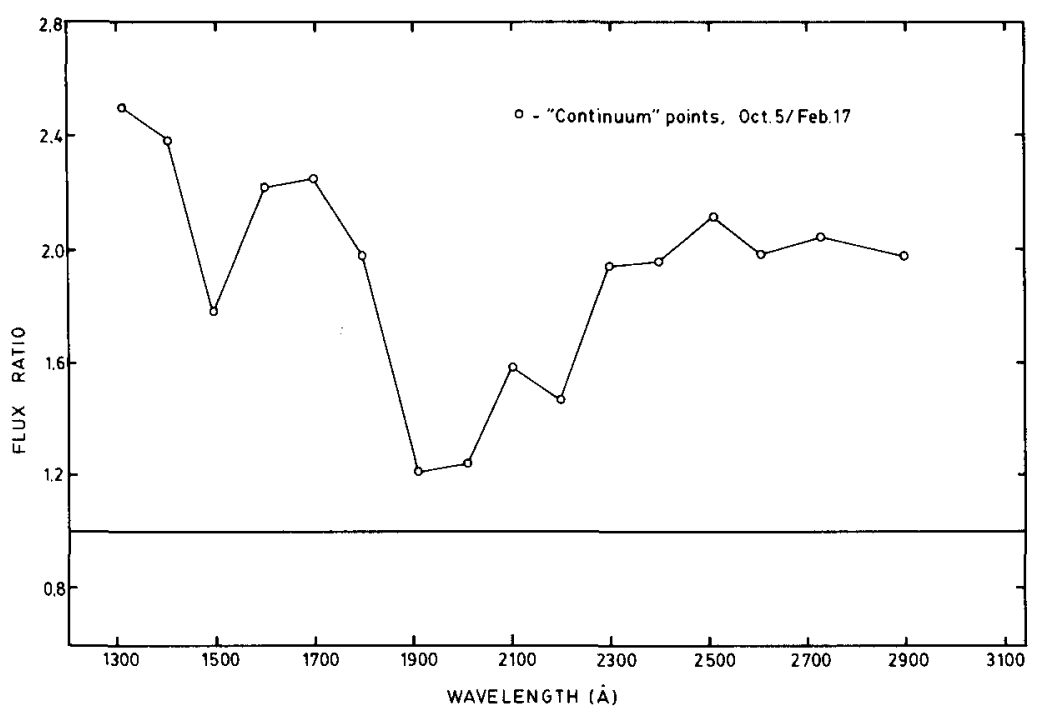

Fig. 2. Ultraviolet continuum variations of HD 207739.

transfer rate of some $10^{-6}$ solar mass per year, it can probably replenish, more or less annually, a layer of plasma of several optical depths (in the ultraviolet) surrounding one of the components or the entire system. On the other hand, rather than a relatively complete replenishment of the emitting plasma, what is occurring may be the injection of sufficient gas to significantly modify the temperature and volume of some regions of the circumstellar or circumbinary plasma envelope. The plasma could be heated by the conversion of kinetic energy to heat through such processes as accretion, shocks or colliding gas streams. The optical thickness of this plasma is significantly larger than the shells, streams, winds, or other gas flows commonly found in interacting binaries. Until a more complete understanding of the processes involved is achieved, we will tentatively call this emitting plasma 'surface' the 'pseudo-photosphere'. This does not imply that it must comprise an extension of the true photosphere or of one of its components.

R Arae was observed with IUE in 1982 over nearly one complete orbital period. Light curves were obtained from IUE's Fine Error Sensor (FES) reading, which is essentially in the blue, and from the continuum flux levels (at various ultraviolet wavelengths) at different phases over one orbital period. The continuum flux levels have been normalized to a standard exposure time. The light curves thus obtained, though relatively crude, exhibit two remarkable characteristics. Figure 3 shows the FES light curve, which is the longest wavelength observed, while Figure 4 shows the 1540 and $1295 \AA$ light curves, the latter being the shortest wavelength at which accurate measurements can be made.

First, there is a secondary minimum near phase 0.6. Second, the depth of this secondary minimum increases toward short wavelengths (as does the primary minimum as may be expected). The appearance of a secondary minimum at phase 0.6 is puzzling. 


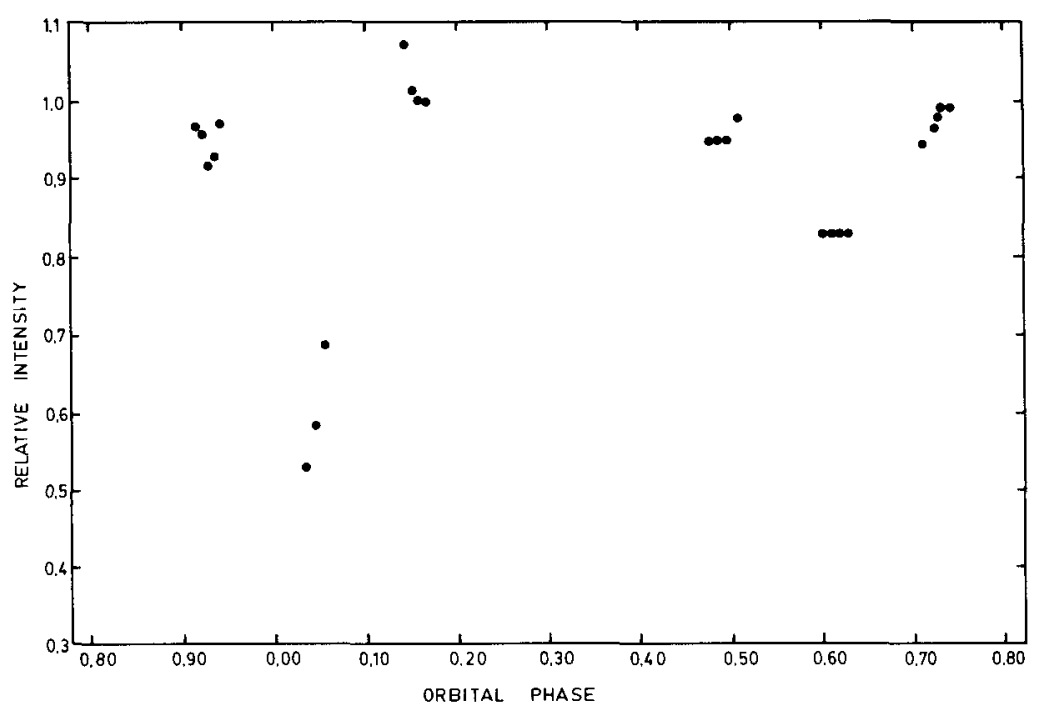

Fig. 3. IUE fine error sensor light curve of $\mathbf{R}$ Arae.

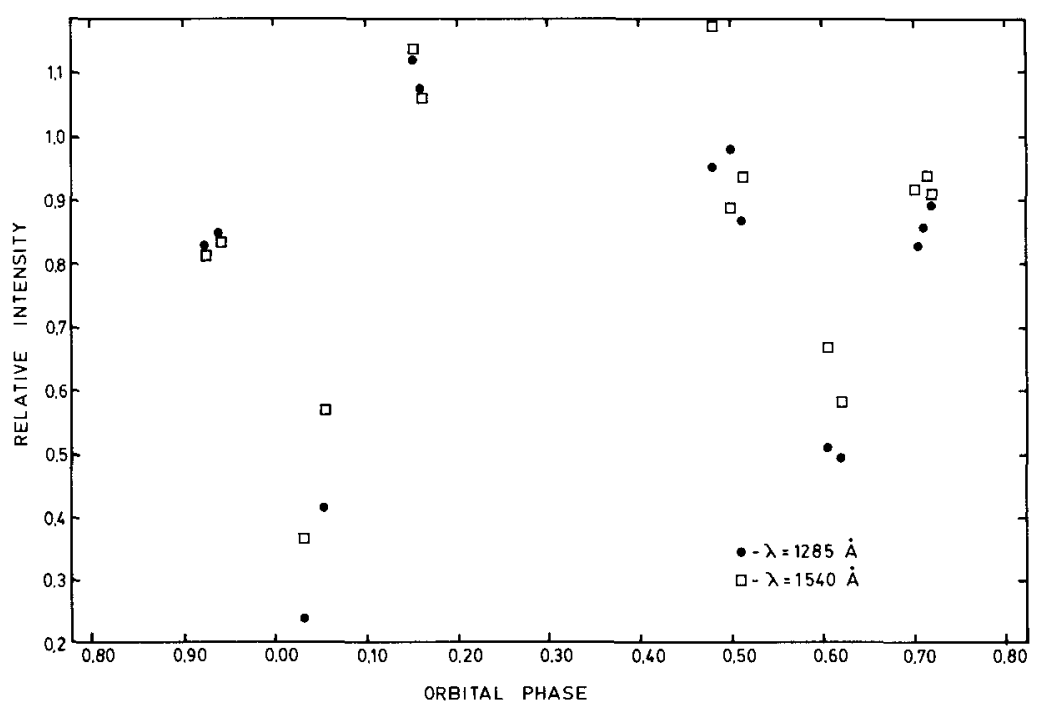

Fig. 4. IUE light curves of R Arae.

Sahade (1952) did not derive the eccentricity for this binary but his velocity curves do not preclude the possibility of some eccentricity in the orbit. In order for the secondary minimum (due to an eclipse) to be displaced to phase 0.6 , the minimum eccentricity required (when the major axis of the ellipse is in the plane of the sky) is about 0.16 . It is, however, not certain that the secondary minimum is simply due to the eclipse of the undetected star by the B8p star. 
The interacting system U Cephei (B7V + G8III-IV, $P=2.5$ days - Batten, 1974) is a much more extensively studied binary. It has a circular orbit with a shallow secondary eclipse occurring at phase 0.5 . However, during an active mass transfer event observed in 1974-75, a secondary minimum was observed at phase 0.6 in the ultraviolet light curves with ANS (Kondo et al., 1978). Perhaps, during an active mass transfer event physical conditions in U Cephei resemble those observed in R Arae (in 1982), which seems to be currently in a continuous state of active mass transfer. If so, the secondary minimum observed at phase 0.6 in $\mathrm{R}$ Arae may not have been an eclipse of the secondary component. In fact, for reasons discussed below, the secondary minimum observed in the ultraviolet is quite unlikely to be an eclipse of the secondary star.

An indication of this is the depth of this eclipse and the dependence of this depth on wavelength. The secondary minimum in $\mathbf{R}$ Arae becomes deeper toward short wavelengths. Blackbody-emitting stars cannot produce such an effect. The eclipse of the cooler star must become shallower at shorter wavelengths. This phenomenon has been observed in two other binaries. It was first reported in $\beta$ Lyrae (B9p + ?, $P=12.9$ days) from photometric data obtained with the Orbiting Astronomical Observatory (OAO-2) by Kondo et al. (1976). The ANS observations of the secondary minimum at phase 0.6 in U Cephei during its active mass transfer event also show that feature to be deepening toward shorter wavelengths; in fact, the entire ANS ultraviolet light curves of U Cephei look very peculiar. As stated earlier, U Cephei has a circular orbit and the secondary minimum observed could not have been the result of an eclipse of the G-type secondary component in that system. In the case of $\beta$ Lyrae, the secondary eclipse, as discussed in the references listed above, shows a very anomalous wavelength dependent behavior but has not been observed to deviate from phase 0.5 .

We suggest that the displaced secondary minimum observed in the ultraviolet for $\mathrm{R}$ Arae and $\mathrm{U}$ Cephei is due to a cooler region in the optically thick plasma which was facing us at phase 0.6. Contrasted to the hotter regions surrounding it, the minimum would become deeper toward short wavelengths. With only two examples, we cannot be certain at the moment whether or not the appearance of a minimum at the same phase, i.e., 0.6 , is indicative of some common process occurring in the mass flow patterns of these systems.

We speculate that R Arae and HD 207739 (and U Cephei during one of its active events) are perhaps in an evolutionary stage just preceding (or following) the short-lived supercritical mass transfer in which $\beta$ Lyrae appears currently to find itself.

\section{Acknowledgement}

One of us (GEM) was partially supported in this research by NASA Grant NSG 5386.

\section{References}

Batten, A. H.: 1974, Publ. Dom. Astrophys. Obs. 11, 191.

Kondo, Y., McCluskey, G. E., and Eaton, J. A.: 1977, Astrophys. Space Sci. 41, 121. 
Kondo, Y., McCluskey, G. E., and Houck, T. E.: 1971, IAU Colloq. 15, 308.

Kondo, Y., McCluskey, G. E., and Wu, C.-C.: 1978, Astrophys. J. 222, 635.

Kondo, Y., McCluskey, G. E., and Wu, C.-C.: 1981, Astrophys. J. Suppl. 47, 333.

McCluskey, G. E. and Kondo, Y.: 1983, Astrophys. J. 266, 755.

Parsons, S. B., Holm, A. V., and Kondo, Y.: 1983, Astrophys. J. 264, L19.

Sahade, J.: 1952, Astrophys. J. 116, 27. 